\title{
Direct-Drive Gas-Cooled Reactor Power System: Concept and Preliminary Testing
}

\author{
S. A. Wright ${ }^{1}$, R. J. Lipinski ${ }^{1}$, T. J. Godfroy ${ }^{2}$, S. M. Bragg-Sitton ${ }^{2}$, M. K. Van \\ Dyke $^{2}$

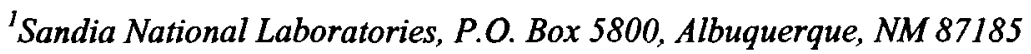 \\ ${ }^{2}$ Marshall Space Flight Center, National Aeronautics and Space Administration, Huntsville, Alabama, 35812 \\ (256) 544-1104,Thomas.Godfroy@msfc.nasa.gov
}

\begin{abstract}
This paper describes the concept and preliminary component testing of a gas-cooled, UN-fueled, pin-type reactor which uses $\mathrm{He} / \mathrm{Xe}$ gas that goes directly into a recuperated Brayton system to produce electricity for nuclear electric propulsion. This Direct-Drive Gas-Cooled Reactor (DDG) is designed to be subcritical under water or wetsand immersion in case of a launch accident. Because the gas-cooled reactor can directly drive the Brayton turbomachinery, it is possible to configure the system such that there are no external surfaces or pressure boundaries that are refractory metal, even though the gas delivered to the turbine is $1144 \mathrm{~K}$. The $\mathrm{He} / \mathrm{Xe}$ gas mixture is a good heat transport medium when flowing, and a good insulator when stagnant. Judicious use of stagnant cavities as insulating regions allows transport of the $1144-\mathrm{K}$ gas while keeping all external surfaces below $900 \mathrm{~K}$. At this temperature superalloys (Hastelloy or Inconel) can be used instead of refractory metals. Super-alloys reduce the technology risk because they are easier to fabricate than refractory metals, we have a much more extensive knowledge base on their characteristics, and, because they have a greater resistance to oxidation, system testing is eased. The system is also relatively simple in its design: no additional coolant pumps, heat exchanger, or freeze-thaw systems are required. Key to success of this concept is a good knowledge of the heat transfer between the fuel pins and the gas, as well as the pressure drop through the system. This paper describes preliminary testing to obtain this key information, as well as experience in demonstrating electrically heated testing of simulated reactor components.
\end{abstract}

\section{INTRODUCTION AND BACKGROUND}

To allow early utilization, system designs must be relatively simple, easy to fabricate, and easy to test using nonnuclear heaters to closely mimic heat from fission. This combination of attributes will allow pre-prototypic systems to be designed, fabricated, and tested quickly and affordably. The ability to build and test units is key to the success of a nuclear program, especially if an early flight is desired. The ability to perform very realistic non-nuclear testing increases the success probability of the system. In addition, the technologies required by a concept will substantially impact the cost, time, and resources required to develop a successful space reactor power system. Some guidelines used in developing and selecting the present concept are:

- Use existing technologies and components where available.

- Build off of the SP-100 and the SNAP-10A experience. (This primarily means use the UN/Re/Nb1Zr fuel pins and use passive water-immersion sub-criticality methods to avoid in-core safety rods).

- Use technologies supported by industry.

- Minimize temperatures where possible by using engineering design approaches.

- Use materials that are robust to chemical and structural challenges (Superalloys and Stainless Steels).

- Select concepts that scale from $\mathrm{kWe}$ to MWe, and

- Use concepts and configurations that are readily ground tested.

The use of these selection criteria and mission requirements led to the selection of a pin-type gas-cooled reactor coupled to CBC (closed Brayton Cycle) power conversion system and dictated the details of the design. 
Figure 1 shows an overall view of the gas-cooled reactor system coupled to two Brayton Cycle systems. The configuration is a direct-drive reactor/Brayton system meaning that the reactor coolant is also the working fluid of the Brayton cycle with no intermediate heat exchanger. The gas coolant is a 70/30 (atom percent) mixture of $\mathrm{He} / \mathrm{Xe}$. The $\mathrm{He} / \mathrm{Xe}$ gas mixture is a good heat transport medium when flowing, and a good insulator when stagnant. Judicious use of stagnant cavities as insulating regions allows transport of the 1144-K gas while keeping all external surfaces below $900 \mathrm{~K}$. At this temperature super-alloys (Hastelloy or Inconel) can be used instead of refractory metals. These metals are readily available, can be easily welded, are very insensitive to corrosion, have well known material properties, and are widely used in the reactor industry. This reduces risk. In addition, because of the greater resistance of these materials to oxidation, this also eases testing of the system. Testing can be performed in a rough vacuum (or even inert gas) rather than the $10^{-8}$ Torr that is needed for $1300-\mathrm{K} \mathrm{Nb} 1 \mathrm{Zr}$ systems.

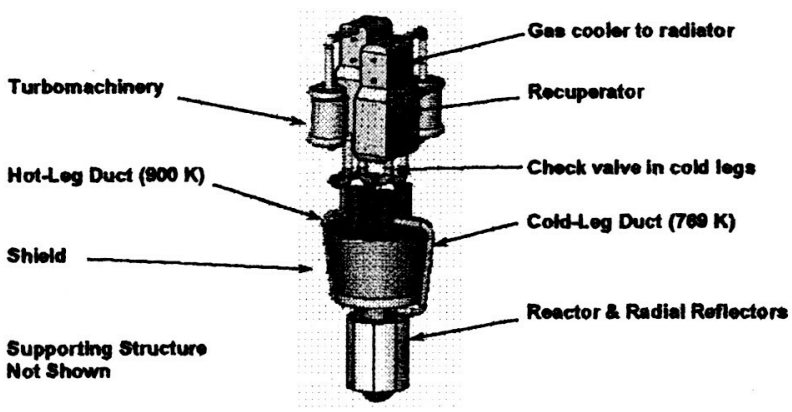

FIGURE 1. Gas Cooled Reactor Concept Illustrating Use of Dual Brayton Cycle Systems.

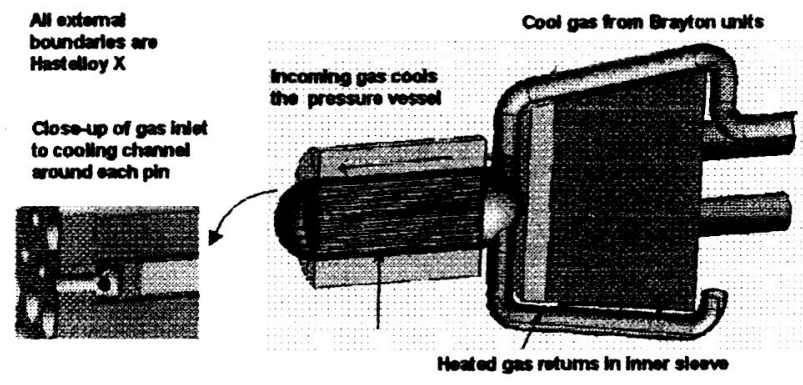

FIGURE 2. Reactor Cutaway Showing Gas Flow Approach Used

to Keep Outer Boundaries Below $900 \mathrm{~K}$.

Figure 2 shows the gas flow configuration that is used to achieve the cooling of the outer pressure boundaries. Cool gas from the Brayton system flows down around the core and cools the pressure vessel before it flows through the core to get heated. As it exits the reactor it enters an internal sleeve in the return duct. Thermal radiation from the outside of the return duct is sufficient to keep the duct surface below $900 \mathrm{~K}$ while the gas in the inner sleeve remains at $1144 \mathrm{~K}$. Only about $10 \mathrm{~kW}$ of thermal power is lost by this approach, which is easily accommodated by the 400-kWt reactor.

Direct coupling to the Brayton system and the exclusive use of superalloy materials for the pressure boundary, permits the direct-drive gas-cooled reactor to be simpler and to avoid a number of challenging design issues. The simplifying benefits include:

- No high temperature heat exchanger

- No transition joint from refractory metal to superalloy

- No high temperature liquid metal pumps (TEM or ALIP)

- No auxiliary radiators for TEM pump thermoelectrics and ALIP waste heat

- No thaw system for the liquid metal or volume accumulators;

- No freeze-thaw concerns

- No liquid metal compatibility (solubility) or environmental, safety, and health concerns

- No gas separators for helium generated by $\mathrm{Li}$

- No exposed refractory metal and $10^{-8}$ Torr testing difficulties

Because of these benefits, the development of the Gas-Cooled Reactor concept could have substantially reduced cost, schedule, and risk compared to other approaches. 
A cross sectional view of the Gas-Cooled Reactor concept is shown in Figure 3 and a more detailed view is in Figure 4. The fuel is $93.15 \%$ enriched $\mathrm{UN}$ that is clad with rhenium-lined $\mathrm{Nb}-1 \mathrm{Zr}$. The pin assembly consists of the fuel pin placed in a core matrix (Hastelloy $\mathrm{X}$ ) to form an annular flow passage (including a Re ribbon-wrap spacer). This approach is similar to the $30 \mathrm{MW}$ HTTR gas cooled reactor that is operating in Japan and that has gas exit temperatures of 1123-1223 K (Saito, 2002). The space reactor contains sufficient rhenium (a neutron poison) to make the reactor subcritical under water immersion accidents without the use of internal shutdown rods. The bottom of the fuel pin has a specially designed end cap that permits a tailored flow control orifice to be inserted into it. The location for this is shown most clearly in Figure 5. The flow for each pin will be tailored to match the power of that pin so that the gas exit temperature for all pins is the same.
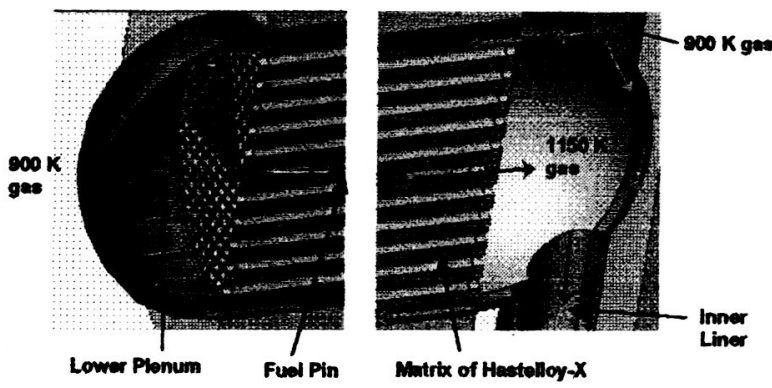

FIGURE 3. Detailed Cutaway of the Reactor Internals.
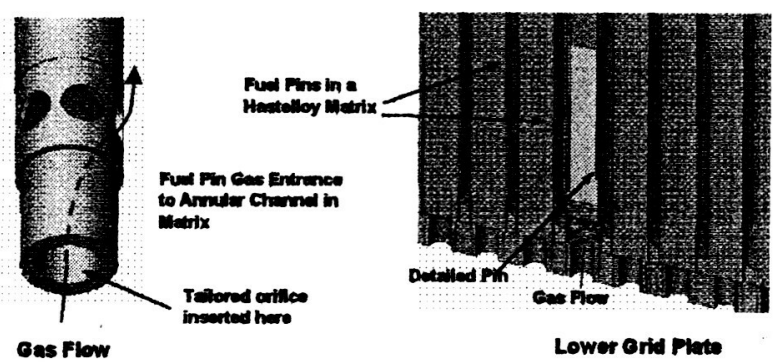

Lower Orid Plate

FIGURE 4. Close-up of the Gas-entrance Region to Annular Channel Around Each Fuel Pin.

\section{Core, Bypass, and Pressure Vessel}

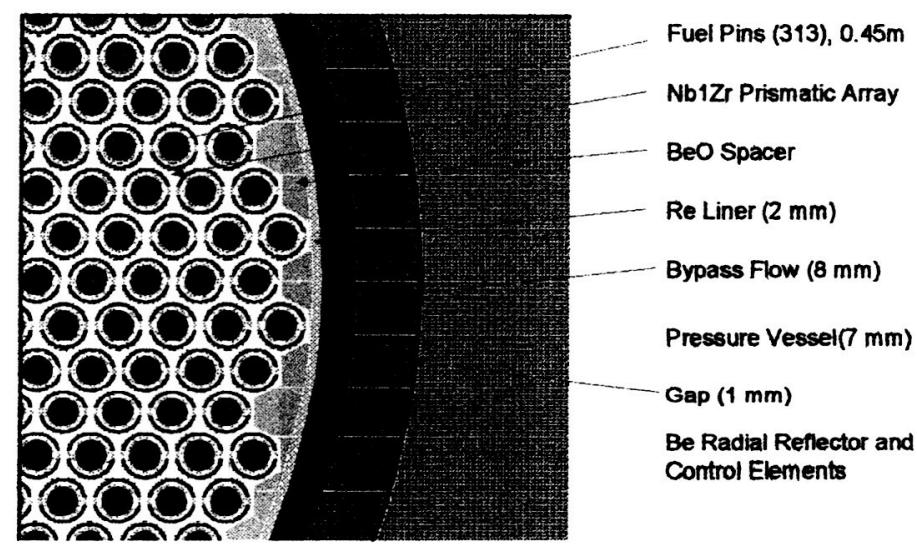

FIGURE 5. Cross Section of the Core Showing Fuel Pins, Annular Channels, Core Matrix, Bypass Flow Region and Pressure Vessel.

The reactor pressure vessel is $0.334 \mathrm{~m}$ in diameter, and the dome-to-dome length at the tip is a little over $1 \mathrm{~m}$. The length of the shield is about $0.7 \mathrm{~m}$ and the shield has a 10 degree half-cone angle. The reactor is shut down by sliding the radial reflectors to open a gap near the center-plane, similar to the SP-100 method of control. Other options for controlling the reactor are angled radial reflector elements (petals), sliding elements, and rotating drums. The design presented in this report uses sliders, however the final selection of the type of control element will be based on the safety merits of the various control methods.

\section{TESTING OBJECTIVES IN THE HPPTS}


The DDG space reactor concept is similar in some ways to a scaled-down version of the 30-MW High Temperature Test Reactor (HTTR) being operated by Japan Atomic Energy Research Institute (JAER) (Saito, 2002). Both use an array of fuel pins in annular flow channels, both use a solid matrix between the pins and annular flow channels to aid in heat removal and temperature stabilization during transients, and both operate at similar gas exit temperatures (about $1150 \mathrm{~K}$ ). The HTTR program has developed heat transfer correlations for fuel pins in annular channels (Takase, 1996) and has developed various techniques for handling the high temperatures. The directdrive reactor will draw upon this database where available, but some of the conditions are different, and techniques developed at JAERI may not be directly applicable. Specifically, the pin dimensions, noble gas mixture, power density, pressure, and flow velocity are different. The electrically heated tests at MSFC will determine whether the HTTR correlations are valid for these new conditions. It will also provide early insights into the testability and fabricability of the direct-drive gas-cooled reactor system.

Building on the successes of the SAFE program which uses "modular testing" to progress through engineering and design issues, the testing of the DDG will occur in phases. The first test article is a reduced power version of a fullscale design, similar to the SAFE-30 test series. This first test article will demonstrate DDR testability by addressing the following issues through non-nuclear testing

- Assembly, operations, and disassembly

- Realistic configuration and matching most operating conditions (pressure, flow rate, core $\Delta T$ )

- Radial power profile matching

- Closed cycle heat transfer to HeAr gas

- Prototypic $\Delta T$

- Benchmark thermal-hydraulic correlations used for a DDG

- Option to couple with Brayton system supplied by Sandia

The specific goals for the first electrically heated testing of the direct-drive gas-cooled reactor concept are as follows:

1. Benchmark thermal-hydraulic correlations that are used in the design of the gas cooled reactor. Because the SAFE heat exchanger $(\mathrm{HX})$ concept is similar in thermal and flow characteristics, both concepts will utilize the same facility saving time and money for testing. Additionally, data from the DDG tests will be beneficial in benchmarking codes and analysis for the SAFE HX as well. DDG testing will:

- Use a module-sized test element from the direct-drive reactor ( 37 pins in a block matrix) with radially dependent power loads and matching flow orifices at the exit end.

- Measure the gas exit temperature at several different channel locations at different flow rates through the channel and to validate the flow resistance correlations. This measurement will be repeated as a function of mass flow rates.

- Vary the total heating rate with time to measure the time dependence of the heat transfer into the gas, and from this determine the heat transfer coefficient into the gas. Results will be compared to the HTTR correlations. This measurement will be repeated as a function of mass flow rates.

- Vary the local heating rate (in one or two rows) to determine the stability of the flow field.

2. Search for evidence of flow vibration in the pins (such as wear, sonic noise, etc) and quantify if possible.

3. Develop and demonstrate techniques for low-cost electrically heated testing and for acceptance testing of flight hardware for gas-cooled reactor systems; identify any potential showstoppers early in testing. Because all of the reactor concepts under consideration consist of large numbers of "small" fuel pins, heater hook-up becomes a major issue. All testable reactor concepts will benefit from this testing. (i.e testing with power delivery capability that simulates a nuclear core, both radially and axially, etc..)

After the 37 pin testing is complete, the follow-on test articles will utilize all of the data and lessons learned to build a higher power core. The testing series is outlined below in Figure 6. 
Data from testing will prove concept capability early in the program rather than relying purely on computational studies which often fail to identify and resolve key issues. This results in a more cost effective approach to the design and development of the system.

\section{TEST ARTICLES DESCRIPTIONS}

The 37-pin, 32-kW, DDG being tested at MSFC is a stainless steel design with 1 inch channels that contain both the heating mechanism and the flow path for heat removal via a gaseous He/Ar mixture. Graphite heaters, demonstrated in the SAFE program, provide the simulated heat from fission using six radial control zones. Since these heaters are designed and fabricated at MSFC, heaters specific to this geometry are fabricated. This heater is inserted into a stainless steel inner tube. The inner tube is wire wrapped to provide a mechanism to obtain directional flow of the gas passing through the channels. A gaseous He/Ar mixture will be used as the heat removal mechanism. The He-/Ar flows along the outside surface of the core, enters a plenum area at the base of the core, flows up through the coolant flow channels, reaches a second plenum area at the top of the core, and exits through a common flow channel to be re-circulated by a gas conditioning system. A gas conditioning system, located outside the vacuum chamber is used to condition the gas in the circulation loop. A pre-heater will be used such that the gas will enter the coolant flow channels from the bottom plenum at a temperature of approximately $655 \mathrm{~K}$. The heater element in the center of each module, used to simulate the heat produced by nuclear fission, will heat the flow to approximately $850 \mathrm{~K}$ by the time it reaches the upper plenum, a $\Delta \mathrm{T}$ of approximately $195 \mathrm{~K}$. The gas entering the flow channels will be at a pressure of approximately $350 \mathrm{psi}$. The gas flow rate through the entire core, consisting of 37 modules, will be $0.1-0.2 \mathrm{~kg} / \mathrm{s}$. Figures 7 through 9 show a graphical representation of the test article.

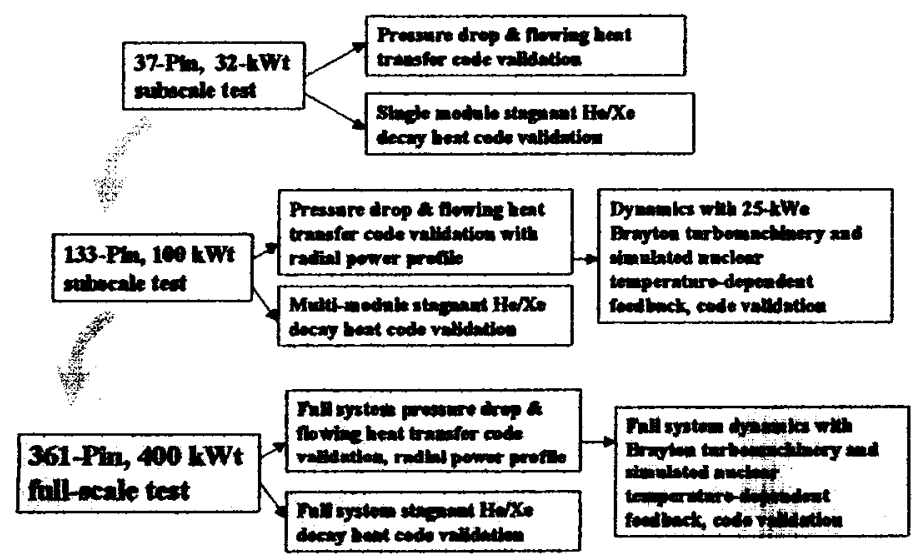

FIGURE 6. Testing Series for a DDR Design.

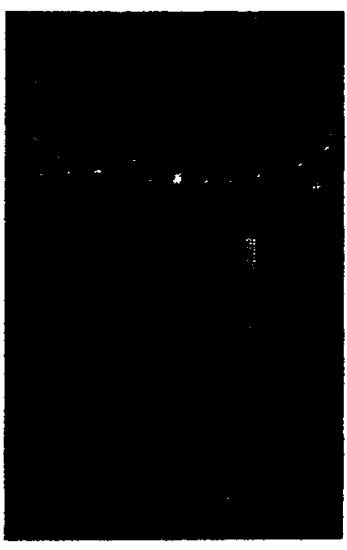

FIGURE 7. DDG Core.

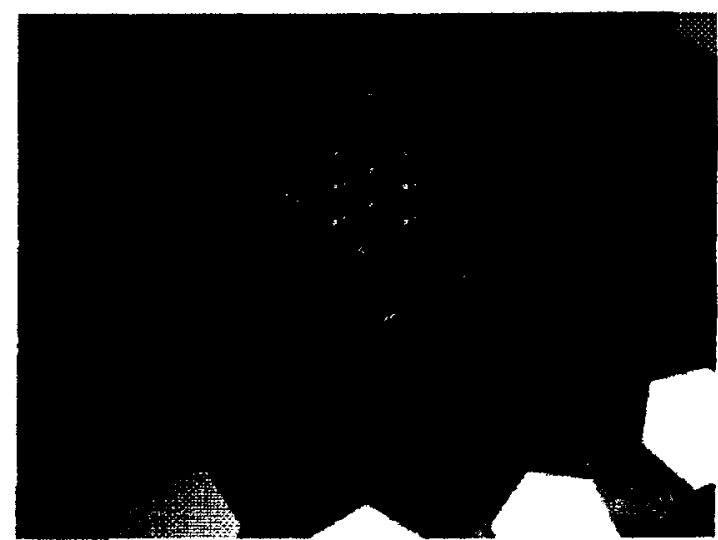

FIGURE 8. Top or Exit End of DDG.

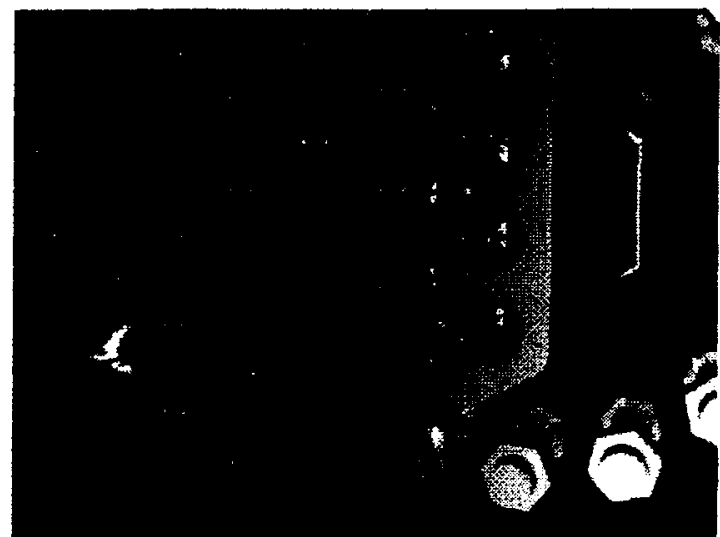

FIGURE 9. Bottom or Inlet End of DDG. 
In order to accomplish the multi-objective program, a comprehensive test matrix is planned. The testing series includes a verification of the vacuum systems, a "cold" and "hot" flow test with GN2, and a hot flow test with a $\mathrm{He} / \mathrm{Ar}$ gas mixture (simulating properties of a gaseous $\mathrm{He} / \mathrm{Xe}$ mixture). Both the test article and the gas conditioning system are currently in fabrication and assembly. Testing is schectuled to being in January 2003.

In addition to a "full" 37-pin test, a one-channel flow characterization test article was fabricated. The primary objectives of the one-channel tests are to characterize the current 37-pin design with respect to coolant flow properties through the core, and to experimentally determine the pressure drop in a single channel. A channel is defined from base (heater connection side) of the core to the top (opposite end) where the gas exits the core (or channel exit). To simplify the single channel flow experiment, the test will not be conducted at the actual temperature and pressure that will be seen in the core demonstrator. Instead, nitrogen gas (replacing the $\mathrm{He} / \mathrm{Ar}$ mixture) will enter the base of the channel at room temperature and will exit the top of the flow channel at ambient pressure. A heater module inside the central tube (representing a fuel pin) will be used to try to match the $\Delta T$ expected for the full core, as determined by type $\mathrm{K}$ thermocouples placed at the inlet and outlet of the flow channel. Matching the expected flow rate for a single channel $(0.1 \mathrm{~kg} / \mathrm{s} / 37$ modules $=2.7 \mathrm{~g} / \mathrm{s})$, the inlet pressure will be measured. The pressure drop will therefore be determined by the difference in the measured pressure at the inlet and the exit (ambient) pressure. For completeness, a range of temperature changes and flow rates will be tested in the single module experiment. Figure 10 shows a representation of a single channel of the DDG. The test article is in fabrication and initial testing is expected to begin in December of 2002.

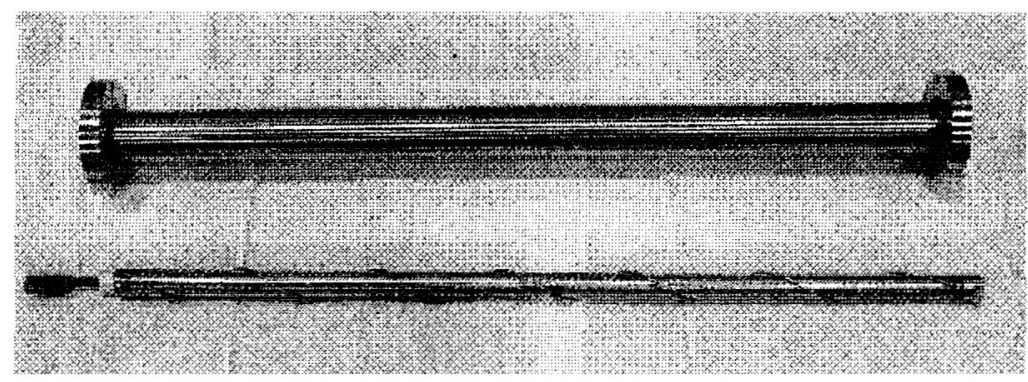

FIGURE 10. Single Flow Channel Hardware

\section{REFERENCES}

Wright, S. A. and Lipinski, R. J., "A Pin-Type Gas-Cooled Direct-Drive Reactor Concept for NEP," in Proceedings of $12^{\text {th }}$ Symposium on Space Nuclear Power and Propulsion, edited by M. El-Genk, AIP Conference Proceedings 324, New York, 1995

Saito, S., "Present Status of the HTTR Project at JAERI," ", IAEA-TECDOC-761, 2002.

Takase, K., "Numerical Prediction of Augmented Turbulent Heat Transfer in an Annular Fuel Channel with Repeated TwoDimensional Square Ribs," Nuclear Engineering and Design, v. 165, pp 225-237, 1996 